\title{
Pretreatment with IFN- $\alpha$ increases resistance to imatinib mesylate in patients with chronic myelocytic leukemia
}

Yin $\mathrm{XIAO}^{1, \#}$, Hui-hua $\mathrm{HU}^{2, \# \text {, Hong-xiang WANG }}{ }^{3}$, Xiao-jian ZHU ${ }^{1}$, Ping ZOU ${ }^{1}$, Zhi-chao $\mathrm{CHEN}^{1}$, Zhao-dong ZHONG ${ }^{1}$, Wei-ming $\mathrm{LI}^{1}$, Yong $\mathrm{YOU}^{1, *}$

${ }^{1}$ Union Hospital, Tongji Medical College, Huazhong University of Science and Technology, Wuhan 430022, China; ${ }^{2}$ Department of Hematology, Centre Hospital, Suizhou 441300, China; ${ }^{3}$ Department of Hematology, Centre Hospital, Wuhan 430014, China

Acta Pharmacologica Sinica (2012) 33: 979-980; doi: 10.1038/aps.2012.43; published online 11 Jun 2012

\section{Dear Editor,}

Chronic myeloid leukemia (CML) is a malignant hematopoietic stem cell proliferative disease driven by BCR-ABL tyrosine kinase, the product of the Philadelphia chromosome ${ }^{[1]}$. Tyrosine kinase inhibitors (TKIs) have revolutionized the treatment of CML. Such molecule directed against BCR-ABL first introduced into clinical practice was imatinib mesylate (IM, Gleevec/Glivec, formerly STI571), which showed excellent efficacy in terms of prolonged major molecular response $(\mathrm{MMR})$ and progression-free survival ${ }^{[2]}$. Replacing hematopoietic stem cell transplantation, IM is currently recommended as the first-line therapy for CML by the National Comprehensive Cancer Network (NCCN) and European Leukemia Net (ELN).

At the pre-TKI stage, IFN-a was the major choice for CML patients who were not candidates for allogeneic stem cell transplantation, and its observed complete cytogenetic response (CCyR) rates were approximately 20\%. Moreover, IFN- $\alpha$ extends the leukemia-free survival (LFS) of patients ${ }^{[3,4]}$. In China, many patients begin with IFN-a treatment instead of IM at diagnosis for economic reasons. Previous studies have shown that the therapeutic efficacy of IM treatment is not affected by prior IFN- $a$ treatment ${ }^{[5,6]}$. The results of our retrospective analysis of $137 \mathrm{CML}$ cases suggest that IFN-a treatment before IM therapy is a risk factor associated with loss of MMR.

In this study, 137 CML outpatients (from June 2008 to May 2010) were retrospectively analyzed. CML diagnosis was confirmed by cytology, immunophenotyping, chromosome $\left(\mathrm{Ph}^{+}\right)$

\footnotetext{
\# These two authors contributed equally to this paper.

* To whom correspondence should be addressed.

E-mail youyongunion@163.com

Received 2011-12-30 Accepted 2012-04-05
}

and BCR-ABL genetic analysis. Among these patients, 122 had CML in the chronic phase (CP), whereas 15 were in accelerated phase or blast phase (AP/BP). A total of $69 \mathrm{CP}$ patients and $8 \mathrm{AP} / \mathrm{BP}$ patients received IFN-a treatment before IM, and the other patients began IM treatment at diagnosis or shortly thereafter. The median period of disease before IM treatment was 3 years for both $\mathrm{CP}$ (1-6.6 years) and AP/BP (1-5 years) patients. IM treatment was continued for $3.5 \pm 1.5$ years for $\mathrm{CP}$ and $3.33 \pm 1.33$ years for $\mathrm{AP} / \mathrm{BP}$ patients. The median followup time was 36 (6-57) months up to May 2010.

The IM dose (administered orally) was initially $400 \mathrm{mg} / \mathrm{d}$ for $\mathrm{CP}$ patients and $600 \mathrm{mg} / \mathrm{d}$ for $\mathrm{AP} / \mathrm{BP}$ patients and was adjusted according to tolerance. Complete molecular remission (CMR) was determined by the absence of BCR-ABL detection. In patients with MMR, the ratio of BCR-ABL and ABL1 was reduced by more than 3 logs after treatment. BCR-ABL transcript levels in patients receiving IM were analyzed every 3 months within the first 12 months of treatment. If MMR was observed, monitoring occurred every 6 months. Quantitative RT-PCR analysis of the kinase domain of the ABL gene from peripheral blood mononuclear cells was performed when the patients lost MMR or did not acquire CMR within 18 months.

The overall 36-month MMR rates for IFN-a-treated and untreated $\mathrm{CP}$ and $\mathrm{AP} / \mathrm{BP}$ patients are shown in Table 1. The MMR rate of IFN-a-treated CP patients (78.3\%) was significantly lower than IFN-a-untreated CP patients (97.3\%). In $\mathrm{AP} / \mathrm{BP}$ patients, the MMR rates of IFN-a-treated and untreated patients (50\% and $60.0 \%$, respectively) did not differ significantly. Furthermore, the MMR rates of patients were analyzed for the association of IFN- $\alpha$ treatment with prolonged disease progression. A chi-square analysis showed that the MMR rate did not differ significantly in $\mathrm{CP}$ patients within the first two years of the disease but was significantly lower in $\mathrm{CP}$ patients 
Table 1. Effect of IFN- $\alpha$ treatment on MMR rate.

\begin{tabular}{|c|c|c|c|c|c|c|}
\hline \multirow{2}{*}{$\begin{array}{l}\text { IFN- } \alpha \\
\text { treat- } \\
\text { ment }\end{array}$} & \multirow[b]{2}{*}{$n$} & \multicolumn{2}{|l|}{ CML-CP } & \multicolumn{3}{|c|}{ CML-AP/BP } \\
\hline & & $\begin{array}{l}\text { Overall } \\
\text { MMR }\end{array}$ & $\%$ & $n$ & $\begin{array}{c}\text { Overall } \\
\text { MMR }\end{array}$ & $\%$ \\
\hline No & 37 & 36 & 97.3 & 5 & 3 & 60 \\
\hline Yes & 69 & 54 & 78.3 & 8 & 4 & 50 \\
\hline
\end{tabular}

who had the disease for more than 2 years $(P=0.0001)$, suggesting relatively reduced IM efficacy in patients with prolonged disease progression. A Cox regression showed that prolonged disease progression was an independent risk factor for IM efficacy $[P=0.006, \operatorname{Exp}(B)=0.625, S E=0.170]$. However, IFN- $a$ was used for all of the patients with disease durations of more than two years, making it impossible to distinguish the effects of disease course and IFN- $a$ treatment on the MMR rate.

In this study, 38 patients $(29 \mathrm{CP}$ and $9 \mathrm{AP} / \mathrm{BP}$ ) were resistant to IM, of which $20 \mathrm{CP}$ and $8 \mathrm{AP} / \mathrm{BP}$ patients (disease course, $3.56 \pm 2.38$ years; average IFN-a treatment duration, $1.2 \pm 0.79$ years) did not acquire CMR within 18 months (primary resistance to IM). A total of 10 patients (9 CP and $1 \mathrm{AP} / \mathrm{BP}$; disease course, $2.09 \pm 1.33$ years; average IFN- $\alpha$ treatment duration, $0.67 \pm 0.43$ years) lost MMR during the treatment (acquired resistance to IM). The disease course of patients with drug resistance (3.56 \pm 2.49 years) was significantly longer than those with persistent MMR $(P=0.0001)$. The IFN-a treatment duration in patients with primary drug resistance ( $3.56 \pm 2.49$ years) was significantly longer than in patients with persistent MMR (1 year) $(P=0.0002)$. In patients with acquired drug resistance, the length of the disease course did not significantly correlate with MMR $(P=0.36)$ or IFN- $\alpha$ treatment duration $(P=0.24)$.

Point mutations in the BCR-ABL gene are the most frequent mechanisms of IM resistance in CML patients and were identified in 38 cases without MMR in our results. These mutations were identified in the kinase region of the ABL fusion gene in $55.3 \%(21 / 38)$ of the patients. Imatinib-resistant BCR-ABL mutants comprising 8 different amino acid locations were identified: P-loop (11 cases, including Y253F/H, E255K/V, G250E, Q252H, and M244V), H396P/R (2 cases), T315I (6 cases) and F359V (2 cases). These mutations were predominantly T315I, Y253H, and E255K/V mutations, in accordance with previous reports ${ }^{[7]}$. The median IFN- $\alpha$ treatment duration for patients with mutations ( $1.45 \pm 0.61$ years) was significantly longer than for patients without mutations $(0.72 \pm 0.61)$ $(P<0.05)$ (Figure 1A). The median pre-IM disease course of the 21 cases with point mutations (1.5 years) was similar to persistent MMR $(P=0.07)$ (Figure 1B). The pre-IM disease course, IM treatment and IFN- $\alpha$ treatment duration did not differ significantly between $\mathrm{AP} / \mathrm{BP}$ and $\mathrm{CP}$ patients. These results suggest that the duration of pre-TKI IFN- $\alpha$ treatment but not the preTKI disease course is associated with ABL point mutations and drug resistance.

In summary, IM therapeutic efficacy correlates with the
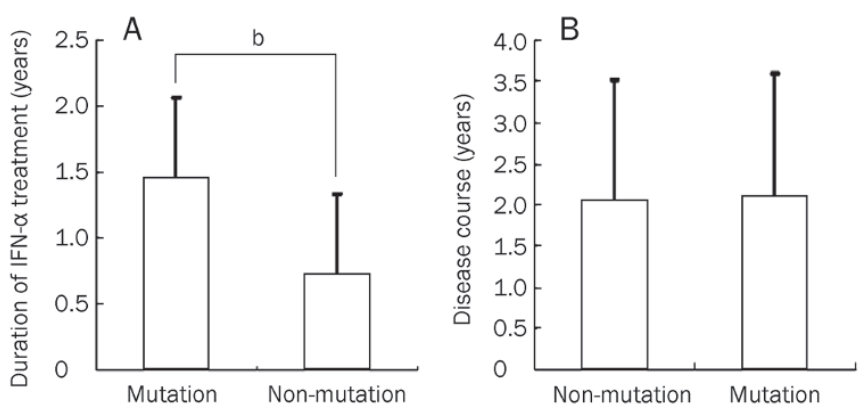

Figure 1. Duration of IFN- $\alpha$ treatment (A) and disease course (B) in patients with or without point mutations. ${ }^{\mathrm{b}} \mathrm{P}<0.05$.

disease course and duration of IFN-a treatment. The duration of pre-IM IFN- $\alpha$ administration in CP patients significantly impacts drug resistance, which is inconsistent with the results of previous studies. This study reveals that IFN-a affects IM resistance in CML patients via increasing the frequency of point mutations. Therefore, it is speculated that early diagnosis and IM administration combined with reduced durations of IFN-a treatment will effectively reduce the drug resistance rate, increase the MMR rate and prolong the leukemia-free survival time.

\section{Acknowledgements}

We thank Shi-ang HUANG and Xiao-qing LI for their skillful technical assistance. We also thank them for kindly providing reagents used in this study. This work was supported in part by grants from the Huazhong University of Science and Technology.

\section{References}

1 Sengupta A, Arnett J, Dunn S, Williams DA, Cancelas JA. Rac2 GTPase deficiency depletes BCR-ABL ${ }^{+}$leukemic stem cells and progenitors in vivo. Blood 2010; 116: 81-4.

2 O'Brien SG, Guilhot F, Larson RA, Gathmann I, Baccarani M, Cervantes $\mathrm{F}$, et al. Imatinib compared with interferon and low-dose cytarabine for newly diagnosed chronic-phase chronic myeloid leukemia. N Engl J Med 2003; 348: 994-1004.

3 Melo JV, Hughes TP, Apperley JF. Chronic myeloid leukemia. Hematology Am Soc Hematol Educ Program 2003; 2003: 132-52.

4 Simonsson B, Hjorth-Hansen H, Bjerrum OW, Porkka K. Interferon alpha for treatment of chronic myeloid leukemia. Curr Drug Targets 2011; 12: 420-8.

5 Kiladjian JJ, Mesa RA, Hoffman R. The renaissance of interferon therapy for the treatment of myeloid malignancies. Blood 2011; 117 : 4706-15.

6 Kujawski LA, Talpaz M. The role of interferon-alpha in the treatment of chronic myeloid leukemia. Cytokine Growth Factor Rev 2007; 18 : 459-71.

7 Branford S, Rudzki Z, Walsh S, Parkinson I, Grigg A, Szer J, et al. Detection of BCR-ABL mutations in patients with $C M L$ treated with imatinib is virtually always accompanied by clinical resistance, and mutations in the ATP phosphate-binding loop (P-loop) are associated with a poor prognosis. Blood 2003; 102: 276-83. 